\title{
Electric Vortex in MHD Flow
}

\section{Manuel Garcia}

RECEIVED

JUN 271995

OSTI

\section{MASTER}

May 1, 1995

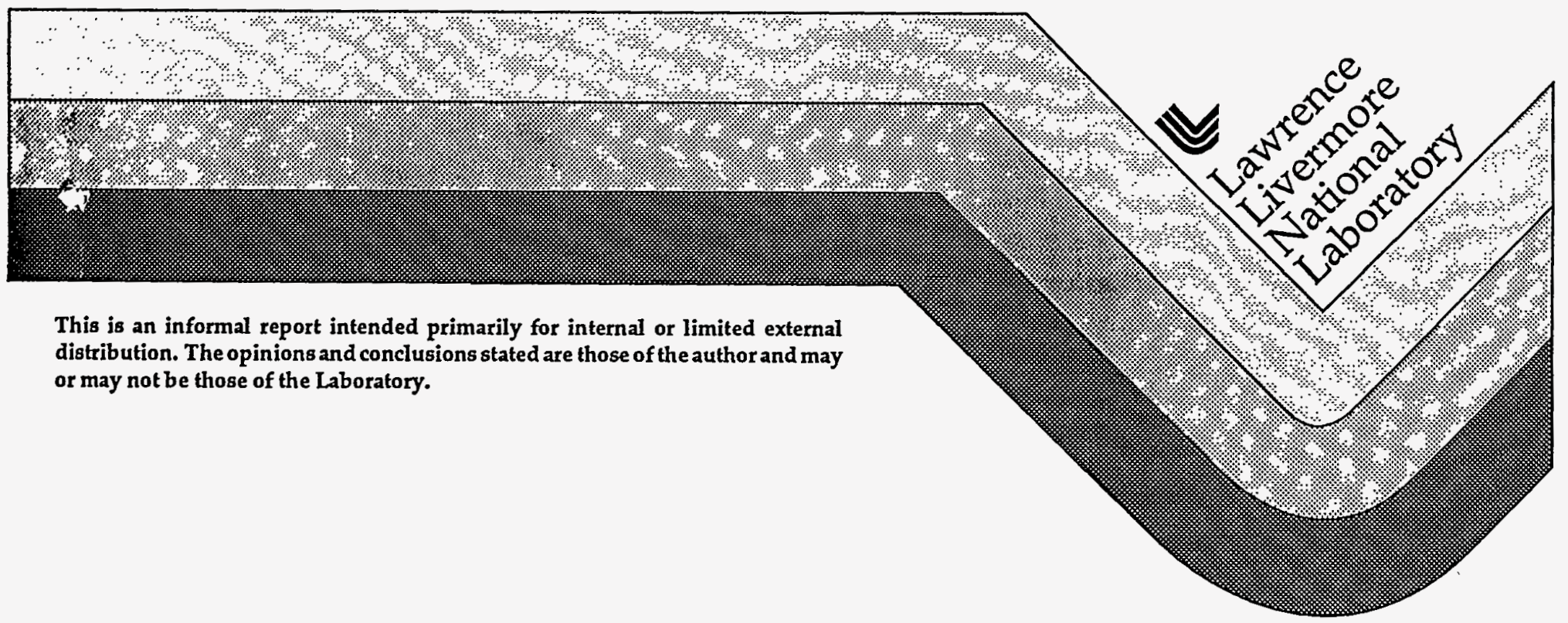




\section{DISCLAIMER}

This document was prepared as an account of work sponsored by an agency of the United States Government. Neither the United States Government nor the University of California nor any of their employees, makes any warranty, express or implied, or assumes any legal liability or responsibility for the accuracy, completeness, or usefulness of any information, apparatus, product, or process disclosed, or represents that its use would not infringe privately owned rights. Reference herein to any specific commercial product, process, or service by trade name, trademark, manufacturer, or otherwise, does not necessarily constitute or imply its endorsement, recommendation, or favoring by the United States Governmient or the University of California. The views and opinions of authors expressed herein do not necessarily state or reflect those of the United States Government or the University of California, and shall not be used for advertising or product endorsement purposes.

This report has been reproduced directly from the best available copy.

Available to DOE and DOE contractors from the Office of Scientific and Technical Information P.O. Box 62, Oak Ridge, TN 37831

Prices available from (615) 576-8401, FTS 626-8401

Available to the public from the

National Technical Information Service

U.S. Department of Commerce

5285 Port Royal Rd,

Springfield, VA 22161 


\title{
Electric Vortex in MHD Flow
}

\author{
Manuel Garcia \\ Lawrence Livermore National Laboratory \\ Livermore, CA, 94551-0808
}

\section{Introduction}

An electric vortex is the circulation of electron space charge about a magnetic field line that is transported by ion momentum. In cold, or low $\beta$ flow the vortex diameter is the minimum length scale of charge neutrality. The distinctive feature of the vortex is its radial electric field which manifests the interplay of electrostatics, magnetism, and motion. ${ }^{1}$

\section{Frozen Flow}

The transport and diffusion of magnetism in MHD is analogous to that of vorticity in classical fluid mechanics. Given:

$$
\begin{aligned}
\partial \mathrm{B} / \partial \mathrm{t} & =\nabla \times(\mathbf{v} \times \mathrm{B})+\nabla^{2} \mathrm{~B} / \sigma \mu_{0}=-\nabla \times \mathbf{E} \\
\mathbf{j} & =\sigma(\mathbf{E}+\mathbf{v} \times \mathbf{B})
\end{aligned}
$$

for magnetic field $B$, plasma velocity $\mathrm{V}$, electric field $\mathrm{E}$, conductivity $\sigma$, and permeability constant $\mu_{0}$, then for $\partial \mathrm{B} / \partial t=0, E=-\nabla \mathrm{V}$, and for $\sigma=\infty, E=-\mathbf{v} \times \mathbf{B}$. Here the velocity and magnetic field lie in equipotential surfaces.

An example is the polarization drift in which a plasma propagates perpendicular to a magnetic field by becoming polarized.

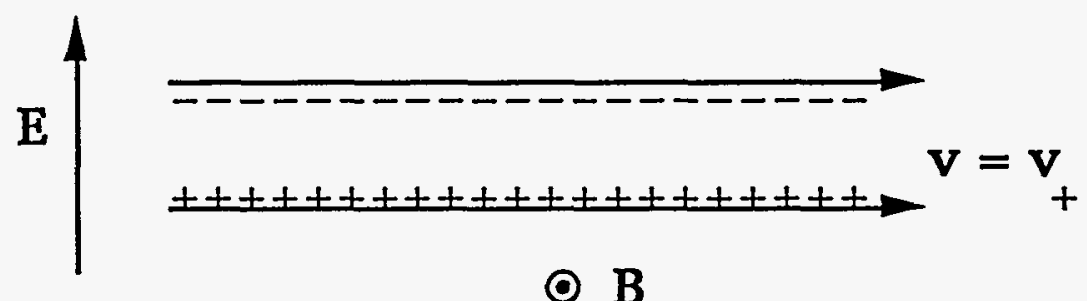

○ B

Figure 1: Polarization Drift

The polarization drift has been observed ${ }^{2,3}$ in plasmas where the dielectric constant satisfies the condition: 


$$
\varepsilon / \varepsilon_{0}=1+\left(\omega_{p+} / \omega_{c+}\right)^{2} \gg\left(m_{+} / m_{e}\right)^{1 / 2}
$$

where $\omega_{\mathrm{p}+}=$ ion plasma frequency, and $\omega_{\mathrm{ct}}=$ ion cyclotron frequency.

Combining condition (3) with the definitions of the Alfvern velocity and number, $a=B /\left(\rho \mu_{0}\right)^{1 / 2}, A=v_{+} / a$, for $\rho=$ plasma mass density, the polarization drift occurs for Alfvén numbers limited by:

$$
A \gg A_{L} \equiv\left(v_{+} / c\right)\left[\left(m_{+} / m_{e}\right)^{1 / 2}-1\right]^{1 / 2}
$$

where $c=$ light speed. The occurrence of the polarization drift can be given from condition (4) as a relationship between the required minimum plasma density given $B$, or maximum magnetic field given $n_{e}$ :

$$
\mathrm{n}_{+\mathrm{L}} / \mathrm{B}_{\mathrm{L}}^{2}=\left(\varepsilon_{0} / \mathrm{m}_{+}\right)\left[\left(\mathrm{m}_{+} / \mathrm{m}_{\mathrm{e}}\right)^{1 / 2}-1\right]
$$

where $\varepsilon_{0}=$ permittivity constant in MKS units, and B is in Tesla.

For protons and electrons specifically the polarization drift occurs for any motion when $A>6.47 \cdot v_{+} / c$, and thus for plasma density $\left(\mathrm{cm}^{-3}\right)$ well above $(47.1 \mathrm{~B})^{2}$, for $\mathrm{B}$ expressed in units of Gauss.

Flows with $A \ll A_{L}$ are magnetically insulated: electrons gyrorotate without propagation parallel to $\mathbf{v}_{+}$across the magnetic field, while ions expend their kinetic energy to form an electrostatic layer.

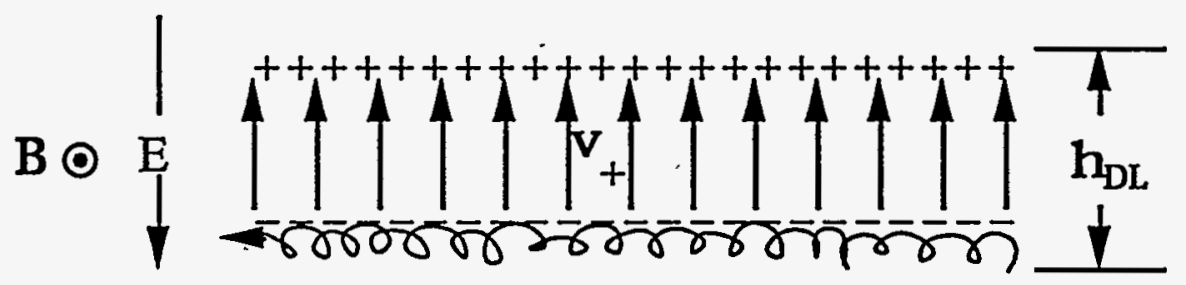

Figure 2: Magnetic Insulation

The thickness of this layer is $h_{D L}=$ Debye length based on the ion kinetic energy. In Figure 2 electron gyroradii are shown much smaller than $h_{D L}$, and the $\mathbf{E} \times \mathbf{B}$ drift of the electron space charge is indicated.

At the transition from magnetic insulation to polarization drift, say by an increase in the ion density injected at velocity $v_{+}$, the laterally drifting electron layer will bunch with a periodicity set by $h_{D L}$. These bunches grow in the direction of ion propagation because the electron layer is an equipotential which cannot be separated further than $h_{D L}$ from its equivalent positive space charge. 
B $\odot$
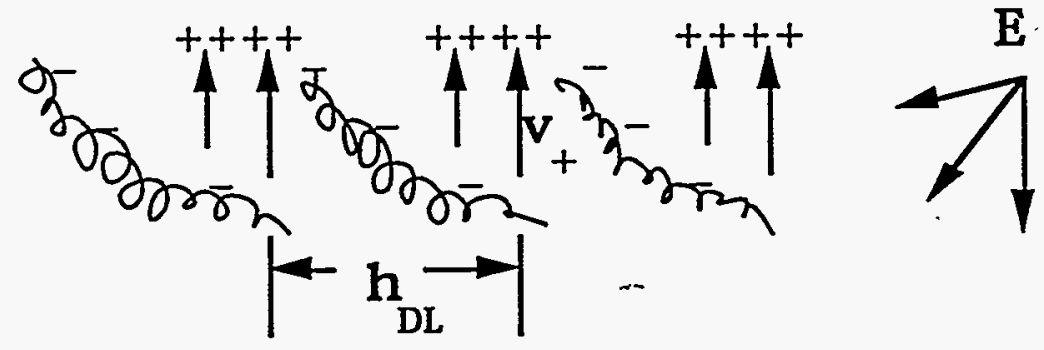

Figure 3: Transition, $A=A_{L}$

The transition from insulation to a polarization drift can be viewed as a rotation of the electric field from an antiparallel direction to a perpendicular one within cells of periodicity $h_{D L}$. In a polarization drift individual electron gyroradii may be minute compared with other dimensions, yet the aggregate electron fluid is carried along with the ion momentum by the intrinsic $\mathrm{E} \times \mathrm{B}$ of charge neutrality. 4

\section{Vortex Structure}

Consider the polarized plasma flow impinging on a stationary, conducting boundary. ${ }^{5}$ Figure 4 shows two schematic views of the resulting vortex structure. The polarization potential across the plasma is shorted by the conducting wall, and electron space charge flows as current loop $-\mathrm{j}$ which transmits the stationary boundary condition to the incoming flow as a decelerating $\mathbf{j} \times \mathbf{B}$ force. The polarization field of the impinging stream becomes radial within the vortex between a positive core and the circulating electronic space charge. The circulation frequency is given by $\omega=v_{+} / r$, where the vortex radius $r$ is set by solid boundaries or in their absence by $h_{D L}$. When $A>1$ the flow is supersonic and the detached $-j$ current sheet ahead of the wall is a shock wave.

As a specific example, for $n_{e}=n_{4}=3 \times 10^{12} \mathrm{~cm}^{-3}$ and proton kinetic energy $E_{K}=6 \mathrm{MeV}$, then $h_{D L}=1 \mathrm{~cm}, B_{L}=3.7$ Tesla, $A_{L}=0.7$, and the polarization drift occurs for $B<B_{L}$ which makes $A>A_{L}$. The resulting vortex has circulation frequency $\omega / 2 \pi=3 \mathrm{GHz}$, and radial electric field $\mathrm{E} \leq \mathrm{E}_{\mathrm{K}} / \mathrm{h}_{\mathrm{DL}}=6 \mathrm{MV} / \mathrm{cm}$.

Alfven has suggested that just as continuum fluid flow is comprised of twisted filaments of vorticity, so the cosmic plasma has a cellular structure with electric vortex filaments as one of its fundamental elements. 5 


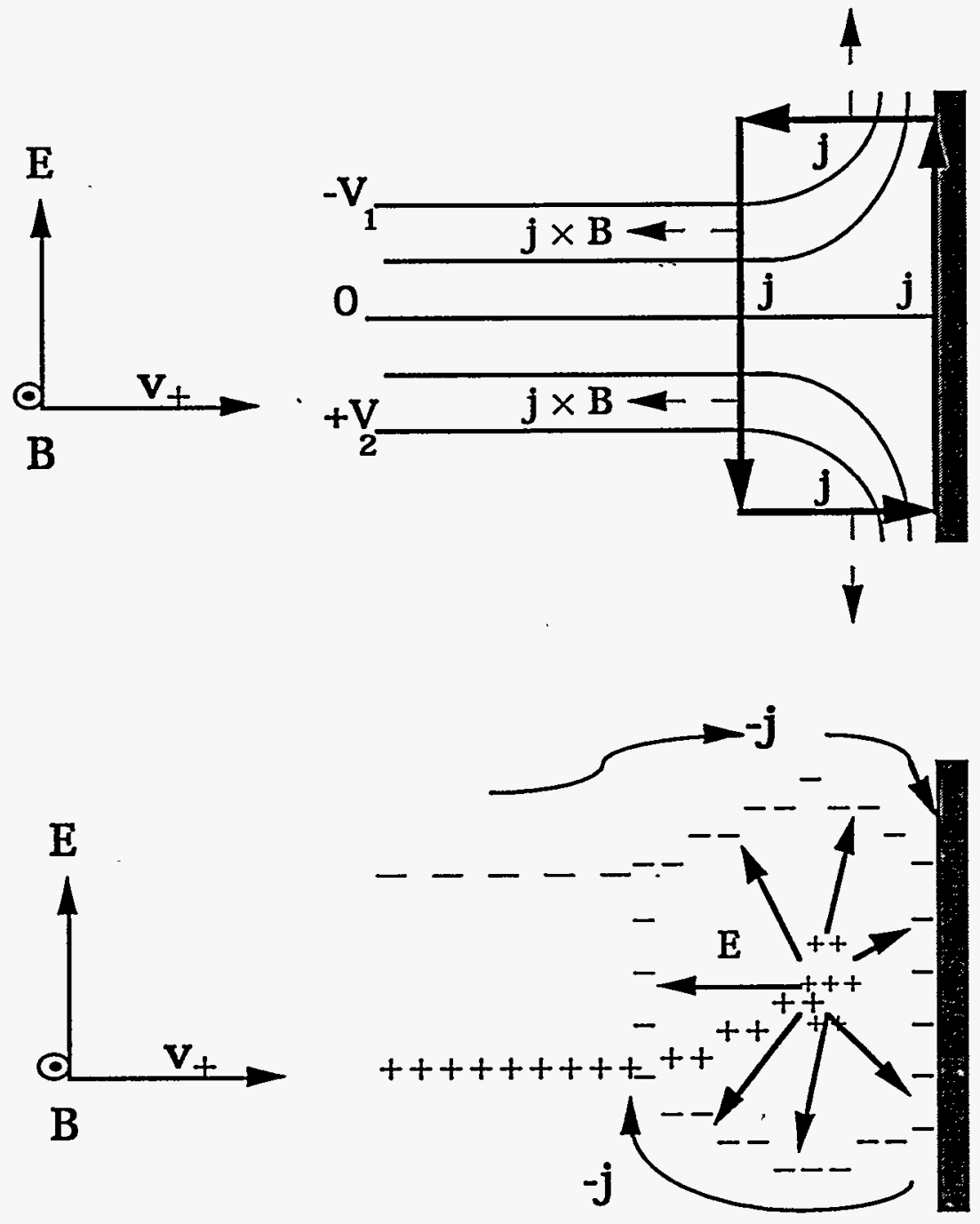

Figure 4: Vortex Structure

\section{Z-Pinch Vortex Sheet}

The sheet of axial current wrapping a cylindrical body of plasma is threaded by azimuthal magnetic field lines which are carried along as vortex cores during a z-pinch compression. The electric field in a vortex will be directly proportional to the rate of $\mathrm{pdV}$ work provided by magnetic compression. This effect is destroyed when vortices dissipate as a result of massive plasma heating.

To quantify these effects an adiabatic, infinite conductivity, inviscid, perfect gas model with azimuthal symmetry was produced. The external magnetic field driving the compression is assumed to have the form: 


$$
B(r, t)=B_{\theta}(r, t) i_{\theta}+B_{z} i_{z}
$$

The trajectory of the outer boundary is found to be:

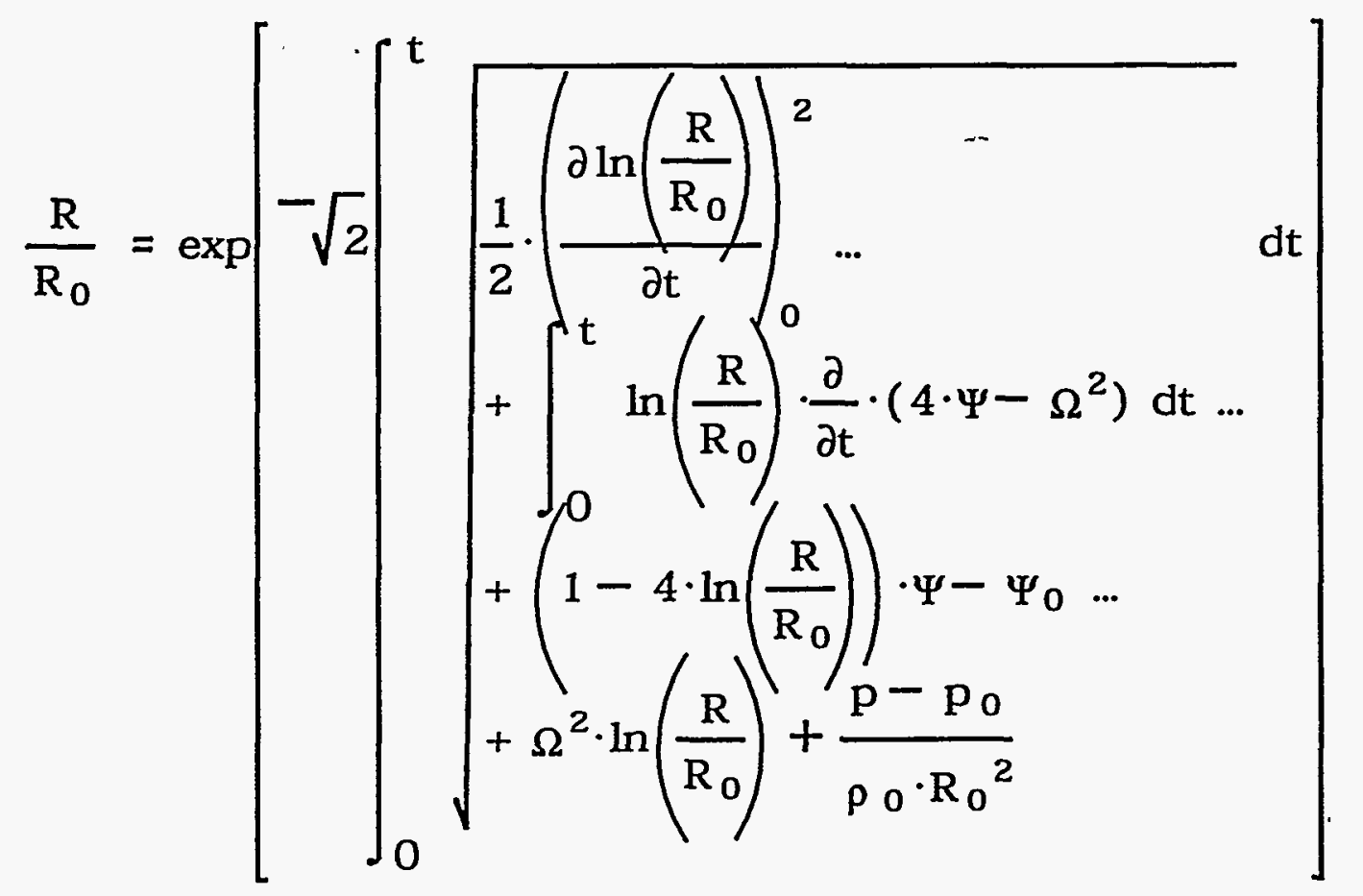

for $t=$ time, $R(t)=$ outer boundary radius, $p(t)=$ exterior pressure, $\rho(t)=$ fluid density, $\Omega(t)=$ solid body rotation of the plasma column, subscripts o denoting initial conditions, and finally:

$$
\Psi(t)=\frac{B_{\theta}(t)^{2}}{2 \cdot \rho_{0} \cdot R_{0}{ }^{2} \cdot \mu_{0}}
$$

for $B_{\theta}(t)$ the magnetic field on the surface $R(t)$. The constant solenoidal field $B_{z}$ is for mathematical convenience in deriving (7). Specific examples are calculated by iteration, eight iterants being calculated with convergence usually in five.

By the very nature of a z-pinch, the Alfvén number in sample calculations remains near unity and well above $A_{L}$. The rapid heating of the plasma quickly undermines the basic assumptions of this model, and computations are terminated when the surface velocity $-\partial \mathrm{R} / \partial \mathrm{t}$ equals the ion thermal speed. The cold vortex layer calculated in this model can have a diameter of microns, circulation frequency over 
$100 \mathrm{GHz}$, and radial electric fields of $20 \mathrm{MV} / \mathrm{cm}$ with a time dependence that mimics that of $\partial(\mathrm{pdV}) / \partial \mathrm{t}$, or the power supplied for compression.

The electric vortex is predominately a low $\beta$, high magnetic

Reynolds Number ( $R e_{M}=\sigma \mu_{0} v L$ ) effect, whereas the $z$-pinch is a rapid evolution towards the opposite extreme of hot, stationary plasma. The model for equation (7) does not address the eventual consumption of the initially cold and thin vortex sheet by the bulk of massively heated plasma. Nevertheless, it may be possible to utilize the vortex electric field in a spectroscopic diagnostic of the dynamics during the initial stages of the z-pinch compression.

Results of a sample calculation follow. Here the starting conditions are: $\mathrm{n}_{\mathrm{e}}=\mathrm{n}_{\mathrm{+}}=2.48 \times 10^{18} \mathrm{~cm}^{-3}$, copper ions, $\mathrm{R}_{0}=2.5 \mathrm{~cm}$ radius, $\Omega=2 \pi \cdot 1000 \mathrm{~Hz}$, and $\mathrm{p}(\mathrm{t})=\mathrm{p}_{0}$. The chosen $\mathrm{B}_{\theta}(\mathrm{t})$ in Tesla, and resulting $\mathrm{J}_{\mathrm{Z}}(\mathrm{t})$ in Amperes are shown below along with the vortex electric field in $\mathrm{V} / \mathrm{cm}$ :
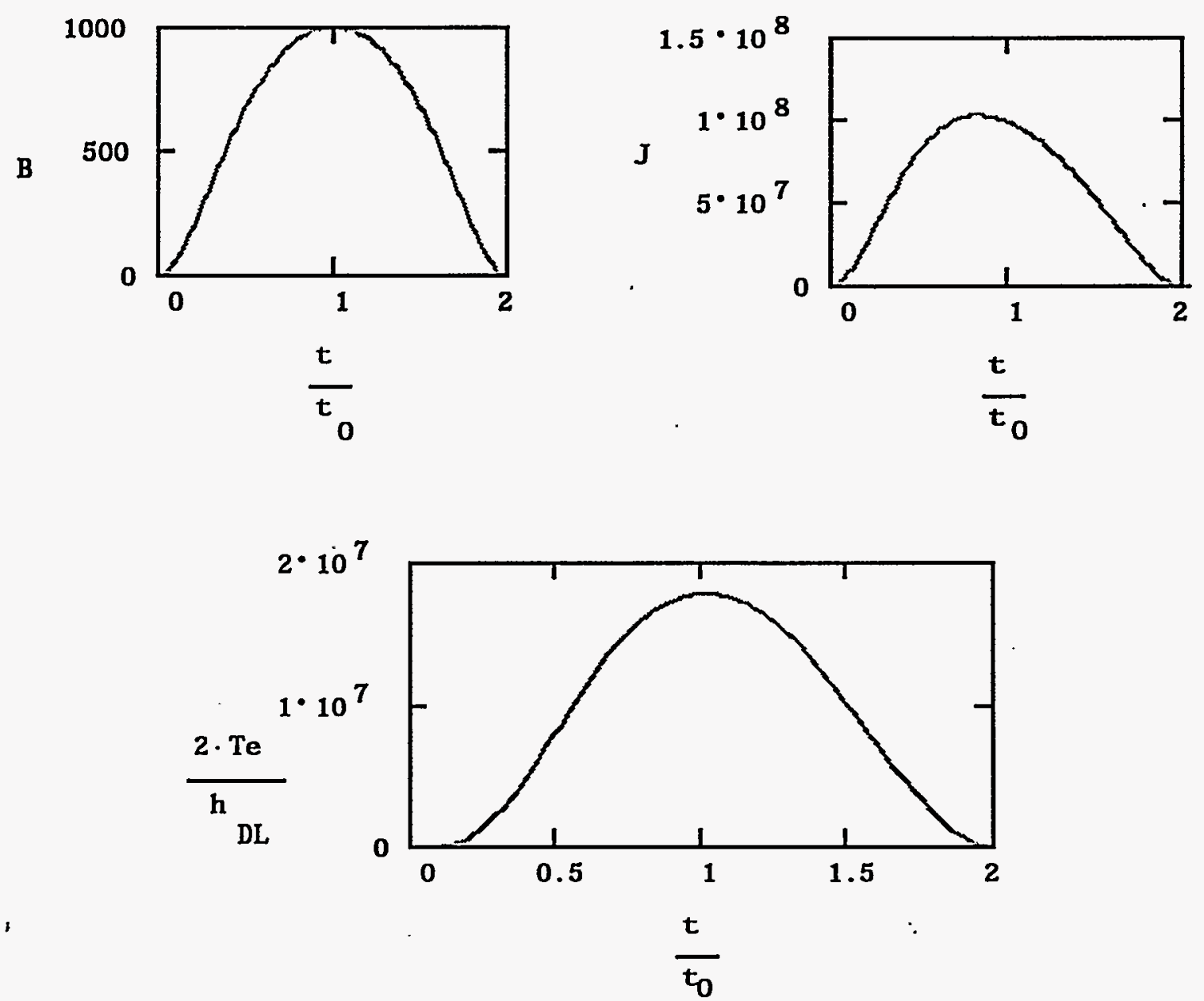

Figure 5: 1000 Tesla @ $t_{0}=5$ ns: $B_{\theta} \quad, J_{z}$, Vortex $E$ field 

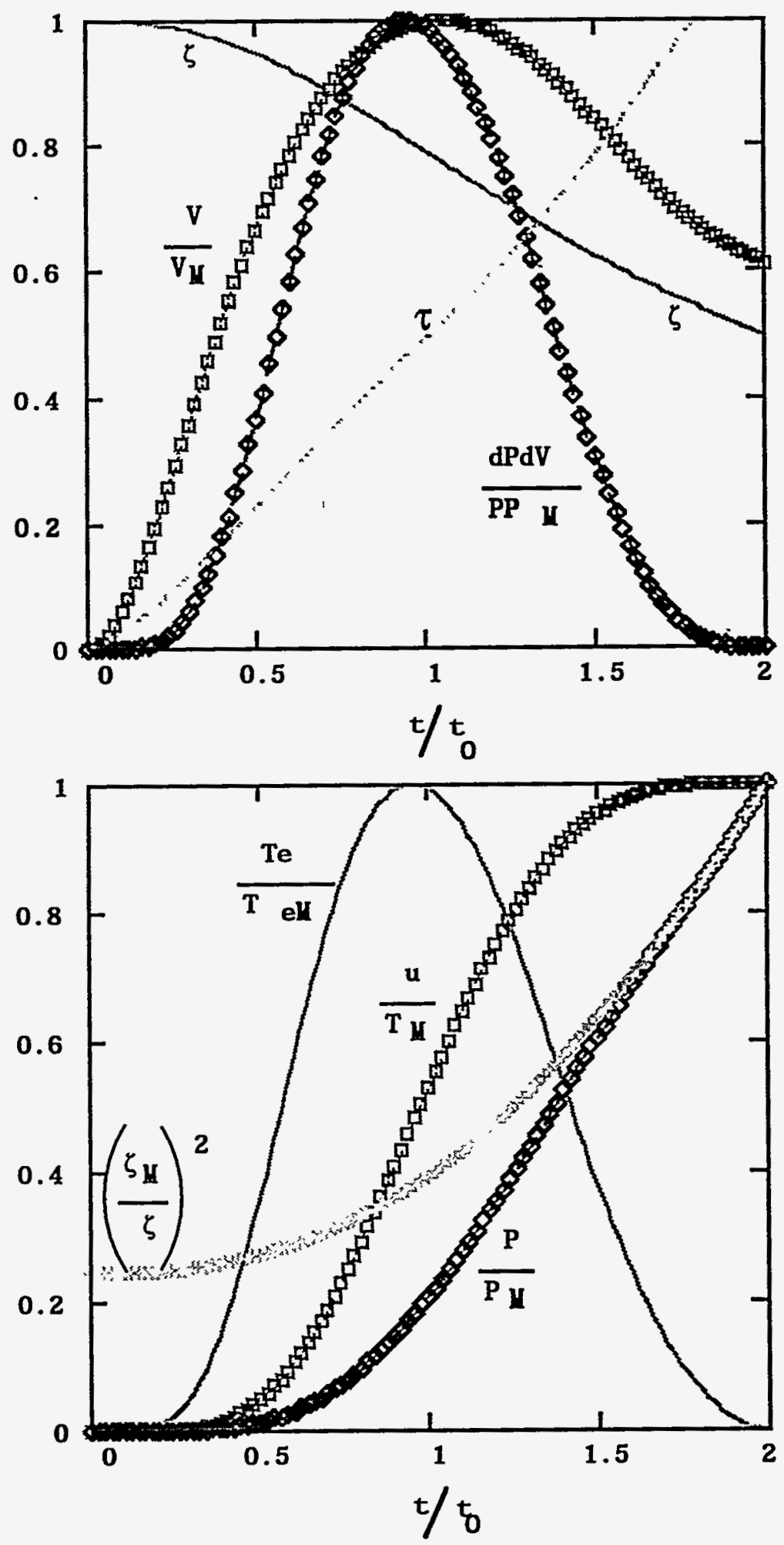

Figure 6: Normalized Profiles: $\zeta$ : radius, V : collapse velocity, $\partial p d V / \partial t:$ power, $\tau:$ ion thermal/collapse speeds, $T_{e}:$ vortex voltage, $\mathrm{u}$ : temperature, $\mathrm{P}:$ pressure, $1 / \zeta^{2}:$ density. See below 
The normalizations used in Figure 6 are: $R_{0}=2.5 \mathrm{~cm},\left(\zeta=R / R_{0}\right)$; $V_{M}=-1.8 \times 10^{6} \mathrm{~m} / \mathrm{s} ; \mathrm{PP}_{\mathrm{M}}=-8.9 \times 10^{16}$ Watts $/ \mathrm{m}$, (per unit length of plasma); $\tau$ is monotonic from 0 to $1 ; T_{e M}=3.4 \mathrm{kV}$, (for vortex radius $\left.\mathrm{h}_{\mathrm{DL}} / 2\right) ; \mathrm{T}_{\mathrm{M}}=472 \mathrm{keV} ; \mathrm{P}_{\mathrm{M}}=6.8 \mathrm{Mbar} ; 1 / \zeta \mathrm{M}^{2}=4.1$, $\left(\rho=\rho_{0} / \zeta^{2}\right.$, density). The characteristics of the vortex layer are shown in Figure 7.
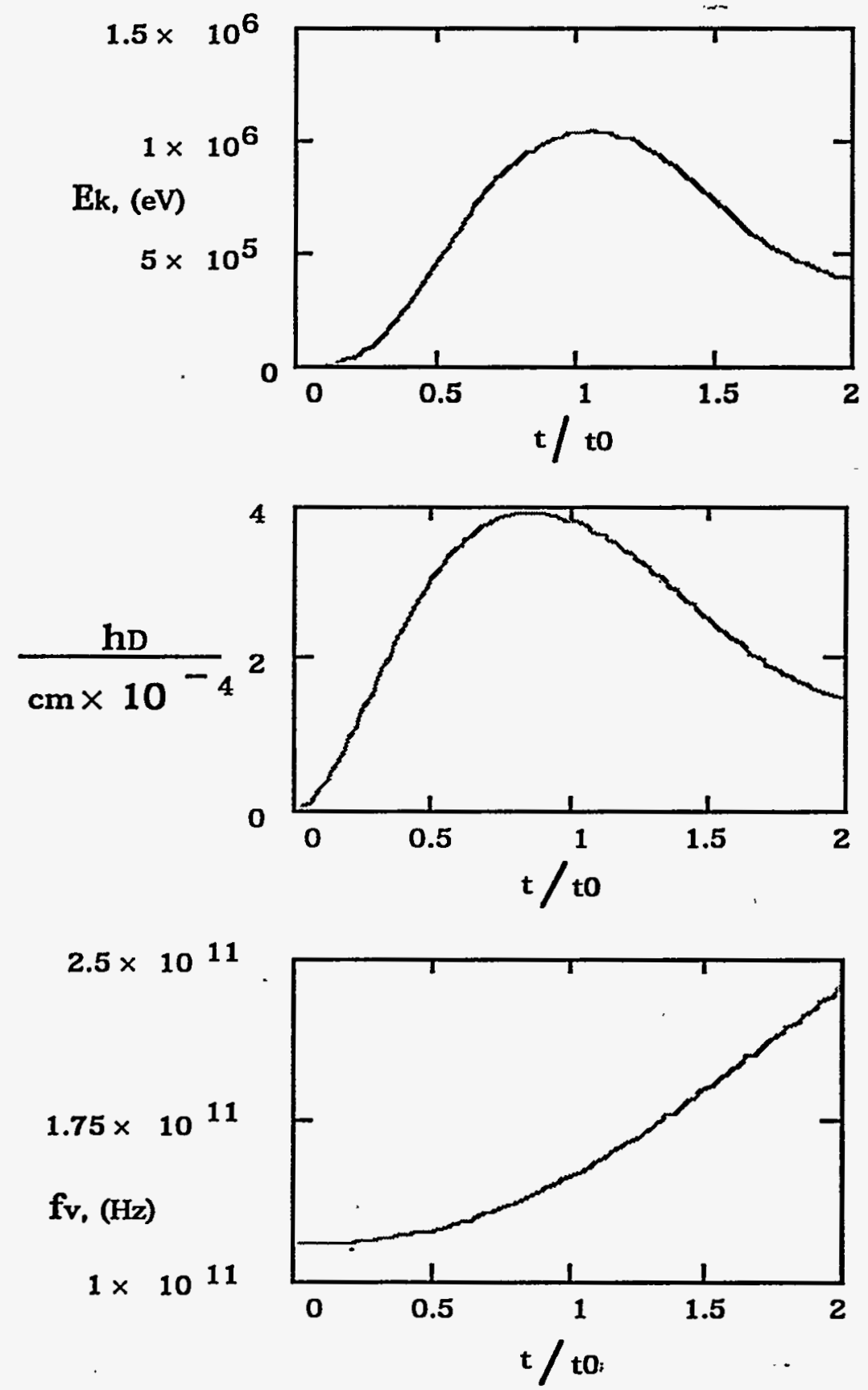

Figure 7: Ion kinetic energy $E_{k}$, vortex radius $h_{D}$, circulation frequency $f_{v}$ 


\section{Conclusions}

An electric vortex is the analog of a Debye shielding length in a magnetized, moving plasma. Three distinctive flow regimes, characterized here as polarization drift, magnetic insulation, and transition, can be delineated by the ratio of the Alfvén number to the parameter $A_{L}$ given in equation (4). The electric field and circulation frequency of electric vortices may be effects accessible for diagnosing experiments. Diagnosis of the early phases of the z-pinch compression will be crucial to understanding the dynamics of the entire process.

\section{Acknowledgments}

I am grateful to Art Toor for his encouragement and the financial support enabling me to attend the 1995 Spring Workshop on Basic Science Using Pulsed Power. The collective work of George Craig, David Kraybill, Joe Pettibone, and Dana Rowley has yielded specific PIC calculations for all the phenomena discussed in Figures 1 through 4. This work was performed under the auspices of the U.S. DOE by LLNL under contract no. W-7405-Eng-48.

\section{References}

1) T. G. Cowling, Magnetohydrodynamics, Adam Hilger, Ltd., New York, 1976, see section 1.6, "Magnetic Rigidity."

2) S. Robertson, H. Ishizuka, W. Peter, N. Rostoker, "Propagation of an Intense Ion Beam Transverse to a Magnetic Field," Phys. Rev. Letts., 47, 7, August 17, 1981, pg. 508.

3) T. Ikehata, K. Kamada, H. Ishizuka, T. Kawabe, S. Miyoshi, "Observation of the Polarization Electric Field in an Intense Neutralized Proton Beam Propagating Undeflectedly Across a Magnetic Field," Phys. Rev. A, 25, 6, June 1982, pg. 3415.

4) S. Humphries, Jr., Charged Particle Beams, John Wiley \& Sons, New York, 1990. See discussions of magnetic insulation in chapter 8, "High Power Pulsed Electron and Ion Diodes." Note that the critical field for insulation defined by Humphries, $\mathrm{Jr}$., $\mathrm{B} *$, is solely a restriction on the extent of the gyroradius of a single electron. As shown here and observed in many ion diode experiments, with sufficient ion current density electrons of arbitrarily small gyroradii easily traverse macroscopic distances of magnetized space.

5) H. Alfvén, Cosmic Plasma, D. Reidel Publishing Co., Boston, 1981. 